\title{
Microbubble Composition and Preparation for High-Frequency Contrast-Enhanced Ultrasound Imaging: In Vitro and In Vivo Evaluation
}

\author{
Verya Daeichin, Member, IEEE, Tom van Rooij, Member, IEEE, Ilya Skachkov, Bulent Ergin, \\ Patricia A. C. Specht, Alexandre Lima, Can Ince, Johan G. Bosch, Member, IEEE, \\ Antonius F. W. van der Steen, Fellow, IEEE, Nico de Jong, Member, IEEE, \\ and Klazina Kooiman, Member, IEEE
}

\begin{abstract}
Although high-frequency ultrasound imaging is gaining attention in various applications, hardly any ultrasound contrast agents (UCAs) dedicated to such frequencies $(>15 \mathrm{MHz})$ are available for contrast-enhanced ultrasound (CEUS) imaging. Moreover, the composition of the limited commercially available UCAs for high-frequency CEUS (hfCEUS) is largely unknown, while shell properties have been shown to be an important factor for their performance. The aim of our study was to produce UCAs in-house for hfCEUS. Twelve different UCA formulations $A-L$ were made by either sonication or mechanical agitation. The gas core consisted of $\mathrm{C}_{4} \mathrm{~F}_{10}$ and the main coating lipid was either 1,2-distearoyl-sn-glycero-3-phosphocholine (DSPC; A-F formulation) or 1,2-dipalmitoyl-sn-glycero-3-phosphocholine (DPPC; G-L formulation). Mechanical agitation resulted in UCAs with smaller microbubbles (number weighted mean diameter $\sim 1 \mu \mathrm{m}$ ) than sonication (number weighted mean diameter $\sim 2 \mu \mathrm{m}$ ). UCA formulations with similar size distributions but different main lipid components showed that the DPPC-based UCA formulations had higher nonlinear responses at both the fundamental and subharmonic frequencies in vitro for hfCEUS using the Vevo2100 high-frequency preclinical scanner (FUJIFILM VisualSonics, Inc.). In addition, UCA formulations F (DSPC-based)
\end{abstract}

Manuscript received September 2, 2016; accepted December 14, 2016. Date of publication December 15, 2016; date of current version March 1, 2017 This work was supported in part by the Center for Translational Molecular Medicine and the Netherlands Heart Foundation, in part by an Innovation Grant of the Dutch Kidney Foundation (14011), in part by NanoNextNL, and in part by a micro and nanotechnology consortium of the Government of the Netherlands and 130 partners.

V. Daeichin, T. van Rooij, I. Skachkov, J. G. Bosch, A. F. W. van der Steen, N. de Jong, and K. Kooiman are with the Department of Biomedical Engineering, Thorax Center, Erasmus MC, 3000 CA Rotterdam, the Netherlands (e-mail: k.kooiman@erasmusmc.nl).

I. Skachkov is now with the Imaging Division, University Medical Center Utrecht, 3508 GA Utrecht, the Netherlands.

A. F. W. van der Steen is also with the Shenzhen Institute of Advanced Technologies, Shenzhen 518000, China, and the Laboratory of Acoustical Wavefield Imaging, Faculty of Applied Sciences, Delft University of Technology, 2628 CJ Delft, the Netherlands.

V. Daeichin and N. de Jong are also with the Laboratory of Acoustical Wavefield Imaging, Faculty of Applied Sciences, Delft University of Technology, 2628 CJ Delft, the Netherlands, and the Netherlands Heart Institute, 3511 EP Utrecht, The Netherlands.

A. Lima and C. Ince are with the Department of Intensive Care Adults, Erasmus MC, 3000 CA Rotterdam, the Netherlands.

C. Ince is also with the Department of Translational Physiology, Academic Medical Center, 1100 DD Amsterdam, the Netherlands.

B. Ergin is with the Department of Translational Physiology, Academic Medical Center, 1100 DD Amsterdam, the Netherlands.

P. A. C. Specht is with the Laboratory of Experimental Anesthesiology, Department of Anesthesiology, Erasmus MC, 3000 CA Rotterdam, the Netherlands.

Digital Object Identifier 10.1109/TUFFC.2016.2640342 and $L$ (DPPC-based) that were made by mechanical agitation performed similar in vitro to the commercially available TargetReady MicroMarker (FUJIFILM VisualSonics, Inc.). UCA formulation $\mathrm{F}$ also performed similar to Target-Ready MicroMarker in vivo in pigs with similar mean contrast intensity within the kidney $(n=7)$, but formulation $\mathrm{L}$ did not. This is likely due to the lower stability of formulation $L$ in vivo. Our study shows that DSPC-based microbubbles produced by mechanical agitation resulted in small microbubbles with high nonlinear responses suitable for hfCEUS imaging.

Index Terms-Contrast agent, contrast-enhanced ultrasound (CEUS) imaging, high-frequency, in vitro, in vivo, microbubble.

\section{INTRODUCTION}

$\mathbf{U}$ LTRASOUND imaging at high frequencies $(\geq 15 \mathrm{MHz})$ enables high-resolution imaging at the price of lower penetration depth, making this technique highly suitable for imaging of small animals [1] and superficial organs in large animals (e.g., skin [2]) and humans (e.g., eye [3] and skin tumors [4]). Alternatively, an endoscopic (e.g., transrectal [5]) or intravascular probe [6] could be used if the organ of interest lies deeper within the body.

Contrast-enhanced ultrasound (CEUS) imaging allows assessment of blood flow to improve diagnosis and monitor therapy. For CEUS, intravenously injected ultrasound contrast agents (UCAs) are needed that consist of gas-coated microbubbles dispersed in saline [7]-[9]. Examples of preclinical high-frequency CEUS (hfCEUS) are tumor angiogenesis imaging [10] and cerebral microvascular hemodynamics assessment in rats [11]. Microbubbles are effective UCAs with a strong resonance structure and inherently nonlinear behavior in response to a time-varying pressure field [10], [11]. These nonlinear oscillations can be present at the subharmonic $(\mathrm{SH})$, fundamental, ultraharmonic, and higher harmonic frequency. The nonlinear signals generated by the microbubbles are utilized in imaging techniques to separate the UCA signal from that of the surrounding tissue [12].

Methods based on the detection of higher harmonics are hindered by artifacts when the excitation frequency is high $(\geq 15 \mathrm{MHz})$, such as nonlinear propagation artifacts [13], [14]. In addition to those artifacts, higher harmonics undergo dramatic attenuation because of their high frequencies, which limits the penetration depth. Nonlinear fundamental contrast imaging is the most common approach 
TABLE I

Production of Microbubbles: Composition of Microbubble Phospholipid Coating, Other Coating Components, Additives, ANd Method of Production; MB = Microbubble

\begin{tabular}{|c|c|c|c|c|c|c|c|c|c|}
\hline \multirow{2}{*}{$\begin{array}{l}\text { UCA } \\
\text { ID }\end{array}$} & \multicolumn{2}{|c|}{$\begin{array}{c}\text { Main coating } \\
\text { component } \\
(\mathrm{mol} \%)\end{array}$} & \multicolumn{3}{|c|}{$\begin{array}{l}\text { Other coating components } \\
(\mathrm{mol} \%)\end{array}$} & \multirow{2}{*}{$\begin{array}{c}\text { Glycerol } \\
(\mathrm{v} / \mathrm{v} \%)\end{array}$} & \multirow{2}{*}{$\begin{array}{l}\text { Propylene } \\
\text { glycol } \\
\text { (v/v \%) }\end{array}$} & \multicolumn{2}{|c|}{ Method of MB production } \\
\hline & DSPC & DPPC & $\begin{array}{l}\text { PEG40 } \\
\text { stearate }\end{array}$ & $\begin{array}{c}\text { DSPE- } \\
\text { PEG2000 }\end{array}$ & $\begin{array}{l}\text { DSPE- } \\
\text { PEG2000 } \\
\text {-biotin }\end{array}$ & & & $\begin{array}{l}\text { Sonication } \\
\text { (time in s) }\end{array}$ & $\begin{array}{l}\text { Vial shake } \\
\text { (time in s) }\end{array}$ \\
\hline$\overline{\mathrm{A}}$ & 59.4 & - & 35.7 & 4.1 & 0.8 & - & - & 60 & - \\
\hline B & 59.4 & - & 35.7 & 4.1 & 0.8 & - & - & 90 & - \\
\hline C & 59.4 & - & 35.7 & 4.1 & 0.8 & - & - & 120 & - \\
\hline D & 59.4 & - & 35.7 & 4.1 & 0.8 & - & - & - & 45 \\
\hline E & 92.4 & - & - & 6.4 & 1.2 & 5 & 5 & - & 45 \\
\hline $\mathrm{F}$ & 92.4 & - & - & 6.4 & 1.2 & 10 & 20 & - & 45 \\
\hline G & - & 59.4 & 35.7 & 4.1 & 0.8 & - & - & 60 & - \\
\hline $\mathrm{H}$ & - & 59.4 & 35.7 & 4.1 & 0.8 & - & - & 90 & - \\
\hline I & - & 59.4 & 35.7 & 4.1 & 0.8 & - & - & 120 & - \\
\hline $\mathrm{J}$ & - & 59.4 & 35.7 & 4.1 & 0.8 & - & - & - & 45 \\
\hline K & - & 92.4 & - & 6.4 & 1.2 & 5 & 5 & - & 45 \\
\hline L & - & 92.4 & - & 6.4 & 1.2 & 10 & 20 & - & 45 \\
\hline
\end{tabular}

for nonlinear hfCEUS imaging [12]. The nonlinear fundamental component suffers less from attenuation, but nonlinear propagation is still a drawback. SH imaging, on the other hand, is free from such artifacts, is less attenuated, and neither generated during propagation in tissue nor scattered by tissue [15]. An SH signal can be achieved with minimum amplitude excitation if the driving frequency is twice the resonance frequency of the microbubbles [16], [17]. To increase the sensitivity of hfCEUS, both the imaging techniques and the UCA design need to be optimized.

The performance of UCAs highly depends on the resonance behavior of a microbubble, which is inversely related to its diameter [17], [18]. Therefore, UCAs with smaller microbubbles can improve the sensitivity of hfCEUS imaging. Next to size, microbubble shell properties have been shown to be an important factor for their performance, mainly in the generation of SH [19], [20]. The lipid composition of the microbubble shell in the commercially available UCAs for hfCEUS is known only for Definity [21], but unknown for MicroMarker [22] and Targestar P-HF [23]. For highfrequency ultrasound molecular imaging, functionalizing the microbubbles is required to target them to the biomarker of interest [24]. Although Target-Ready MicroMarker provides streptavidin linkage [25], no UCA is commercially available to provide covalent coupling of the ligand to the microbubble shell, limiting flexibility in choice of ligands. In-house produced UCAs could overcome these disadvantages.

Based on a preliminary experiment we conducted before [26], the aim of our study was to produce lipid-coated UCAs in-house with high nonlinear response for hfCEUS. Based on microbubble size, a resonance frequency can be predicted [18], but the actual response still depends on the shell microstructure, as we previously showed for the main lipids used in commercially available UCAs [20], [21], [27]: 1,2-distearoyl-sn-glycero-3-phosphocholine (DSPC) or 1,2-dipalmitoyl-sn-glycero-3-phosphocholine (DPPC). We therefore studied 12 different UCA types with the aim to produce microbubbles with a size similar to Definity [21] and
MicroMarker [22]. UCAs were either produced by sonication, i.e., the most common UCA production method [28], or mechanical agitation, i.e., the method by which Definity is prepared [21]. In addition, we compared the UCA compositions we [29] and others [30], [31] have used throughout the years, and quantitatively evaluated the performance of the in-house produced UCAs to Target-Ready MicroMarker in vitro and in vivo with hfCEUS using the most commonly used high-frequency ultrasound imaging platform (Vevo2100, FUJIFILM VisualSonics, Inc., Toronto, ON, Canada).

\section{Materials AND Methods}

\section{A. Ultrasound Contrast Agent Preparation}

All UCAs were produced in phosphate-buffered saline (PBS; Invitrogen, Groningen, the Netherlands) as the aqueous medium. UCAs were either produced by probe sonication at $20 \mathrm{kHz}$ with a Sonicator Ultrasonic Processor XL2020 at setting 10 (Heat Systems, Farmingdale, NY, USA) as previously described [29], [32] for 60,90 , or $120 \mathrm{~s}$ or by mechanical agitation for $45 \mathrm{~s}$ using a Vial Shaker (BristolMyers Squibb Medical Imaging, Inc., North Billerica, MA, USA). UCAs with a $\mathrm{C}_{4} \mathrm{~F}_{10}$ (F2 Chemicals, Preston, U.K.) gas core and different phospholipid coating formulations (see Table I) were made. The main phospholipid component for all UCA formulations was either DSPC (P6517), purchased from Sigma-Aldrich, Zwijndrecht, the Netherlands (formulations A-F) or DPPC (850355), purchased from Avanti Polar Lipids, Alabaster, AL, USA (formulations G-L). The other coating components were polyoxyethylene (40) stearate (PEG-40 stearate; P3440; Sigma-Aldrich), 1,2-diste-aroylsn-glycero-3-phosphoethanolamine-N-carboxy (poly-ethylene glycol) (DSPE-PEG2000; 880125; Avanti Polar Lipids), and 1, 2-distearoyl-sn-glycero-3-phosphoethanolamine-N-biotinyl (polyethylene glycol) (DSPE-PEG2000-biotin; 880129; Avanti Polar Lipids).

Coating formulations A-D and G-J were identical to our previously produced UCAs made by sonication for $10 \mathrm{~s}$ 
(number weighted mean $\sim 4 \mu \mathrm{m}$ ) [29]. The UCAs made by vial shaking either contained no glycerol and no propylene glycol (formulation D and J) as reported in [33] and [34] or contained $5 \% \mathrm{v} / \mathrm{v}$ glycerol (818709, Merck Millipore, Merck, Amsterdam, the Netherlands) and 5\% v/v propylene glycol (82280, Sigma-Aldrich) (formulation E and K) as reported in [31], or 10\% v/v glycerol and $20 \% \mathrm{v} / \mathrm{v}$ propylene glycol (formulation F and L) as reported in [30]. Our UCA formulations $\mathrm{E}, \mathrm{F}, \mathrm{K}$, and $\mathrm{L}$ contained only two different lipids, which is typical for in-house produced UCAs made by vial shaking [30], [35]-[37]. We chose DSPE-PEG2000 in addition to the main lipid DSPC or DPPC because this lipid can be utilized for targeting, whereas the PEG40-stearate cannot [24]. The molar ratio of DSPC or DPPC to (DSPEPEG2000 + DSPE-PEG2000-biotin) was kept identical to UCA formulations A-D and G-J.

UCAs produced by sonication (formulations A-C and G-I) were put in 5-mL glass serum bottles (223738; Wheaton, Millville, NJ, USA), topped with $\mathrm{C}_{4} \mathrm{~F}_{10}$ gas, and closed with a rubber stopper (Z166065; Sigma-Aldrich) and aluminum cap (224193-01; Wheaton). Before the experiments, UCAs made by sonication were washed three times by centrifugation at $400 \mathrm{~g}$ for $1 \mathrm{~min}$ (Heraeus Biofuge, Thermo Scientific, EttenLeur, the Netherlands) to remove the excess lipids. For the vial shaking method, $1 \mathrm{~mL}$ of the solution (lipid concentration $0.4 \mathrm{mg} / \mathrm{mL}$ for formulations $\mathrm{D}-\mathrm{F}$ and $\mathrm{J}-\mathrm{L}$ ) was pipetted in a 2-mL glass screw top vial (5182-0714; Agilent Technologies, Santa Clara, CA, USA), topped with $\mathrm{C}_{4} \mathrm{~F}_{10}$ gas, and closed with a screw cap (5182-0717; Agilent Technologies).

Target-Ready MicroMarker UCA was prepared from the ready kit (FUJIFILM VisualSonics, Inc.) according to the manufacturer's instructions: the lyophilisate cake was reconstituted with $1 \mathrm{~mL}$ of $0.9 \%$ sodium chloride solution. The microbubbles in this UCA consist of a phospholipid shell encapsulating a $\mathrm{C}_{4} \mathrm{~F}_{10} / \mathrm{N}_{2}$ gas core [22]. All UCAs were produced or reconstituted not more than a few hours before the experiments. Size distributions of the UCAs were measured using a Coulter Counter (Multisizer 3, Beckman Coulter, Mijdrecht, the Netherlands). A 20- $\mu \mathrm{m}$ aperture tube was used, allowing quantification of particle diameters between 0.4 and $12 \mu \mathrm{m}$ using a linear spacing between the 256 channels. Measurements were repeated three times for each UCA to obtain the mean microbubble diameter, size distribution, and concentration. Polydispersity of the UCAs was calculated by assessing the SPAN, which illustrates the width of the distribution, using $(\mathrm{d} 90 \%-\mathrm{d} 10 \%) / \mathrm{d} 50 \%$ where $\mathrm{d} 10, \mathrm{~d} 50$, and d90 are the microbubble number weighted diameters below which $10 \%, 50 \%$, and $90 \%$ of the cumulative amount of number weighted microbubbles is found. Data are presented with standard deviations (SDs).

\section{B. In Vitro hfCEUS Imaging and Quantification}

A schematic of our experimental setup is depicted in Fig. 1. The 10-mm diameter thin shell cylindrical tube, made of polypropylene film backing and coated with a water-based acrylic adhesive having a total thickness of $52 \mu \mathrm{m}$ (Tesa 4024 PV 2), was mounted in a water tank with its center at the focus $(18 \mathrm{~mm})$ of the imaging probe. The tube was filled

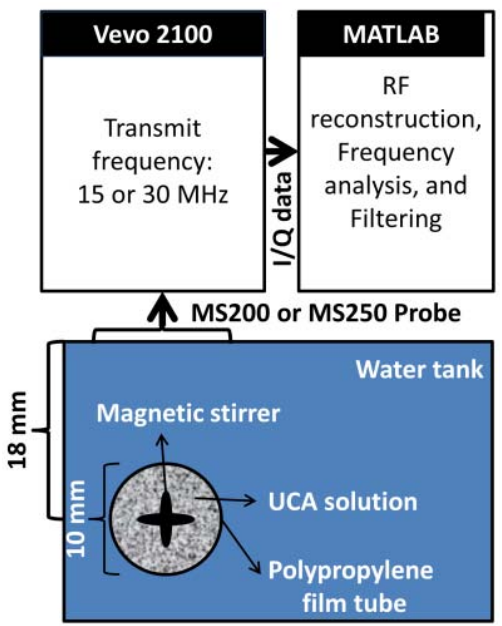

Fig. 1. Experimental setup for the in vitro characterization of the UCA.

with $15 \mathrm{~mL}$ air-saturated PBS. UCA dilutions were pipetted in the tube and mixed gently using a magnetic stirrer in order to have a homogeneous suspension. For the first B-mode scan, the UCA concentration was $5 \times 10^{4}$ microbubbles $/ \mathrm{mL}$. Next, the effect of the UCA concentration on SH imaging was tested for selected UCAs using two concentrations: $8 \times 10^{6}$ and $4 \times 10^{5}$ microbubbles $/ \mathrm{mL}$, hereafter referred to as high and low concentration, respectively. All measurements were conducted within $10 \mathrm{~min}$ after pipetting the UCA suspensions into the tube. For each new UCA or dilution, the tube was washed with distilled air-saturated water, filled with air-saturated PBS, and placed in the same location in the water tank.

We used a high-frequency preclinical ultrasound scanner operated at 15 or $30 \mathrm{MHz}$, with two linear array transducers [MS200 probe $(15 \mathrm{MHz})$ and MS250 probe $(30 \mathrm{MHz})$, FUJIFILM VisualSonics, Inc.]. The wide beamwidth setting was chosen in order to have a low more uniform transmit pressure over depth in the tube [12]. To study the scattering properties of all 12 UCA formulations, the MS200 probe was used at $15-\mathrm{MHz}$ transmit frequency at $1 \%$ transmit power and one cycle pulse duration in B-mode. On the selected UCA formulations (C, I, F, L, and Target-Ready MicroMarker), SH imaging was performed with the MS250 probe. This probe has a center frequency of $22.5 \mathrm{MHz}$ and a $-6-\mathrm{dB}$ two-way bandwidth of $70 \%(15-30 \mathrm{MHz})$ [12], therefore suitable for SH imaging if transmitting at $30 \mathrm{MHz}$. The selection of the $\mathrm{SH}$ imaging parameters such as transmit frequency $(30 \mathrm{MHz})$, power $(10 \%$ corresponding to a peak-to-peak pressure of $\sim 200 \mathrm{kPa}$ ), pulse sequence (pulse inversion [12]), and length (20 cycles for better separation of the SH component in the frequency domain) were based on a previous study in which these parameters were optimized for SH imaging [15]. The focus of the MS250 probe was set at $18 \mathrm{~mm}$.

For all experiments, radiofrequency (RF) signals were reconstructed from I/Q samples and further postprocessed offline using MATLAB (The MathWorks, Natick, MA, USA). Three regions of interest (ROIs) were selected within the tube containing the UCA: ROI 1 was centered at $15 \mathrm{~mm}$; ROI 2 at $18 \mathrm{~mm}$ (focal point); and ROI 3 at $21 \mathrm{~mm}$. Two hundred RF 
lines were averaged in the frequency domain for analysis of UCA responses at the fundamental $(15$ or $30 \mathrm{MHz})$ and $\mathrm{SH}$ $(15 \mathrm{MHz})$ frequencies within each ROI.

RF data were digitally bandpass filtered around SH frequencies in the frequency domain, with a fifth-order Butterworth filter. The -6 -dB frequency cutoff for the SH filter was chosen from 13 to $17 \mathrm{MHz}$. One should note that, in our analyses, the signals at the fundamental frequency $(30 \mathrm{MHz})$ correspond to the nonlinear behavior of the UCAs at that frequency and not the linear backscattered signal. This is because the analyses were performed on the RF signals reconstructed from the pulse inversion sequence, which removes the linear components of the backscattered signal at the fundamental frequency [38].

\section{In Vivo hfCEUS Imaging and Quantification}

The animal protocol was approved by the animal ethics committee of the Erasmus MC (EMC3379 142-14-01) and conducted in strict accordance to the National Guidelines for Animal Care and Handling. After overnight fasting with free access to water, the female pigs (crossbred Landrace $x$ Yorkshire, 3-4 months of age) of approximately $30 \mathrm{~kg}(n=4)$ were premedicated with an intramuscular injection of tiletamine $(5 \mathrm{mg} / \mathrm{kg})$, zolazepam $(5 \mathrm{mg} / \mathrm{kg})$ (Zoletil, both Virbac Laboratories, Carros, France), and xylazine $(2.25 \mathrm{mg} / \mathrm{kg})$ (Sedazine 2\%, AST Farma BV Oudewater, the Netherlands). Anesthesia was maintained with a combination of intravenous infusion of midazolam $(1.5 \mathrm{mg} / \mathrm{kg} / \mathrm{h}$, Actavis, New Jersey, USA), ketamine $(5 \mathrm{mg} / \mathrm{kg} / \mathrm{h}$, Alfasan, Woerden, the Netherlands), sufentanil ( $4 \mu \mathrm{g} / \mathrm{kg} / \mathrm{h}$, Sufenta Fort, Janssen Pharmaceuticals Ltd., USA), and rocuronium bromide ( $4 \mathrm{mg} / \mathrm{kg} / \mathrm{h}$, Fresenius Kabi, Germany) through an ear vein cannula. The animals were ventilated through an endotracheal tube $(7.0 \mathrm{Fr})$, placed in the trachea via midline cervical tracheostomy in a volume controlled mode (Servo 300, Siemens-Elema, Solna, Sweden) with a fraction of inspired oxygen of 0.40 , a frequency to achieve normocapnia, and a positive end-expiratory pressure of $5 \mathrm{~cm} \mathrm{H}_{2} \mathrm{O}$. Surgery via the right flank was performed to expose the right kidney. hfCEUS imaging was performed by manually injecting a $1 \mathrm{~mL}$ UCA bolus in the jugular vein followed by a $10 \mathrm{~mL} 0.9 \%$ sodium chloride flush. The performance of selected in-house produced UCA formulations ( $\mathrm{F}$ and $\mathrm{L}$ ) was compared with that of TargetReady MicroMarker. The order in which they were injected was random. The nonlinear hfCEUS measurements were recorded using the Vevo2100 equipped with an MS250 transducer $[18-\mathrm{MHz}$ transmit frequency, 10 frames/s, $10 \%$ power, $\sim 400 \mathrm{kPa}(\mathrm{MI}<0.1)]$. The Vevo2100 uses the amplitude modulation pulse sequence in the nonlinear contrast imaging mode, which mainly detects the nonlinear fundamental component of the UCA [12]. Immediately after injection of the UCA into the catheter, the ventilation of the animal was paused to minimize movement due to breathing. After 25-30 s, the measurement had been completed and the ventilation was turned on again. Cine loops of side-by-side B-mode and nonlinear contrast mode images were stored as lossless DICOM images for further offline analysis using MATLAB. First, correction for tissue motion in the imaging plane was applied as described
TABLE II

UCA Formulations: MEAN \pm SD MICROBUBbLE CONCENTRATION, Size, AND SPAN

\begin{tabular}{ccccc}
\hline \hline UCA & $\begin{array}{c}\text { CONCENTRAT } \\
\text { ION }\left(10^{9} \text { PER }\right. \\
\text { ID })\end{array}$ & $\begin{array}{c}\text { number } \\
\text { weighted } \\
\text { diameter }(\mu \mathrm{m})\end{array}$ & $\begin{array}{c}\text { volume } \\
\text { weighted } \\
\text { diameter }(\mu \mathrm{m})\end{array}$ & SPAN \\
\hline A & 1.9 & 1.9 & 6.4 & 2.3 \\
B & 1.6 & 2.0 & 5.9 & 1.9 \\
C & 2.3 & 1.9 & 5.7 & 2.7 \\
D & 6.9 & 0.6 & 5.6 & 0.7 \\
E & 16.7 & 0.8 & 3.4 & 1.4 \\
F & 7.0 & 1.1 & 4.6 & 2.6 \\
G & 2.5 & 2.1 & 5.4 & 1.9 \\
H & 1.9 & 2.0 & 4.8 & 2.1 \\
I & 3.0 & 1.7 & 4.6 & 1.0 \\
J & 1.2 & 0.6 & 7.5 & 1.9 \\
K & 4.7 & 0.8 & 5.8 & 2.2 \\
L & 10.0 & 0.9 & 2.8 & \\
Target- & 1.0 & 1.5 & 4.0 & \\
Ready & & & & \\
Micro & & & & \\
Marker & & & &
\end{tabular}

previously [39], [40]. Briefly, the motion pattern of tissue in the field of view was extracted from the B-mode images and applied to the contrast mode images to correct for the motion in the field of view. Three ROIs were chosen for every DICOM recording at a depth of $0.5-5,5-9$, and $9-13.5 \mathrm{~mm}$. For each ROI, all pixel intensities were summed and normalized to the area of the ROI. The intensity in each frame was obtained to construct a time-intensity curve (TIC), the frames with the maximum intensity were detected, and the mean intensity and $\mathrm{SD}$ of this frame and the five frames before and after this frame were calculated. In addition, alternative ROIs were chosen to compare contrast enhancement in the artery with that in the microcirculation where no clear vascularity was visible. The ratio between the intensities in these ROIs was used to quantify the ability to discriminate the blood vessels from the peripheral enhancement.

\section{Statistics}

The ratios for the arteries and microvasculature of TargetReady MicroMarker and UCA formulation $F$ were tested for significance using paired-samples student's $t$-tests, after first ensuring that the data were normally distributed using Shapiro-Wilk normality tests. A $p$-value $<0.05$ was regarded as indicating significance.

\section{RESULTS}

\section{A. Ultrasound Contrast Agent Preparation}

After production, all UCA formulations appeared white as shown in supporting Fig. 1(a) and (b), indicating that UCAs could be produced with all 12 formulations. UCA formulations $\mathrm{D}$ and $\mathbf{J}$ were least white. In addition, both these formulations had the smallest layer of microbubbles at the top of the aqueous solution in the vial after leaving the vials on the bench for $2 \mathrm{~h}$ [supporting Fig. 1(c) and (d)], suggesting a lower amount of microbubbles in these formulations.

The number weighted size distribution of UCA formulations A-L and Target-Ready MicroMarker are shown in Fig. 2; 

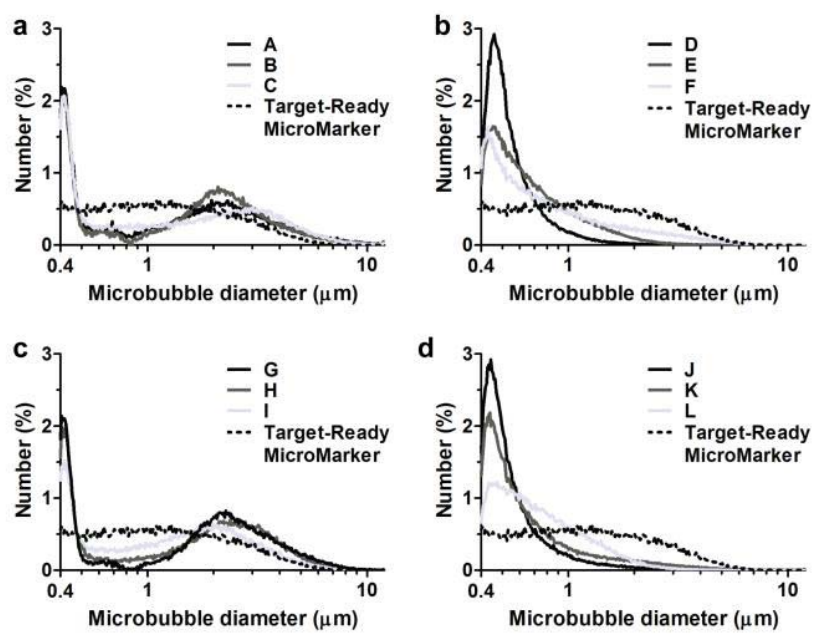

Fig. 2. Number weighted size distributions of (a) DSPC-based UCAs made by sonication (UCA type A, B, and C), (b) DSPC-based UCAs made by vial shaking (UCA type D, E, and F), (c) DPPC-based UCAs made by sonication (UCA type G, H, and I), and (d) DPPC-based UCAs made by vial shaking (UCA type J, K, and L) all compared with Target-Ready MicroMarker.

the volume weighted size distribution is shown in supporting Fig. 2. None of the A-L type UCA formulations had a number weighted mean diameter, volume weighted mean diameter, or size distribution identical to that of TargetReady MicroMarker. UCA formulations A-C and G-I made by sonication [Fig. 2(a) and (c)] contained higher amounts of large microbubbles than Target-Ready MicroMarker. All the UCAs produced by sonication had two distinct peaks in their size distribution $(0.4-0.5$ and $2-3 \mu \mathrm{m})$. Longer sonication times did not alter the mean number weighted diameter much, as shown in Table II. The volume weighted diameter decreased upon longer sonication times, while the concentration of microbubbles increased. UCA formulations D-F and J-L made by vial shaking resulted in smaller microbubbles [Figs. 2(b) and 2(d); Table II] than when microbubbles were made by sonication. DSPC or DPPC as main coating resulted in microbubbles of similar mean number weighted diameters for both UCA production methods (Table II). The highest microbubble concentration was found in UCA formulation E. Microbubbles with the smallest mean number weighted diameter were found in UCA formulation D and J, while this was UCA formulation $\mathrm{L}$ for microbubbles with the smallest mean volume weighted diameter, all of which were produced by vial shaking. The highest mean number weighted diameters were found in formulations $\mathrm{B}$ and $\mathrm{H}$, and the highest volume weighted diameter was found in UCA formulation J. The UCA formulation with the smallest SPAN was formulation D, while formulation $\mathrm{F}$ had the largest SPAN.

\section{B. In Vitro hfCEUS}

As shown in Fig. 3, different ultrasound scattering intensities of UCA formulations A-L were observed at $15 \mathrm{MHz}$ in vitro. The error bars in Fig. 3 represent the variation in B-mode signal intensity within the ROI (the entire cross section of the cylindrical tube containing the UCA). For both the DSPC-based and DPPC-based UCA formulations,

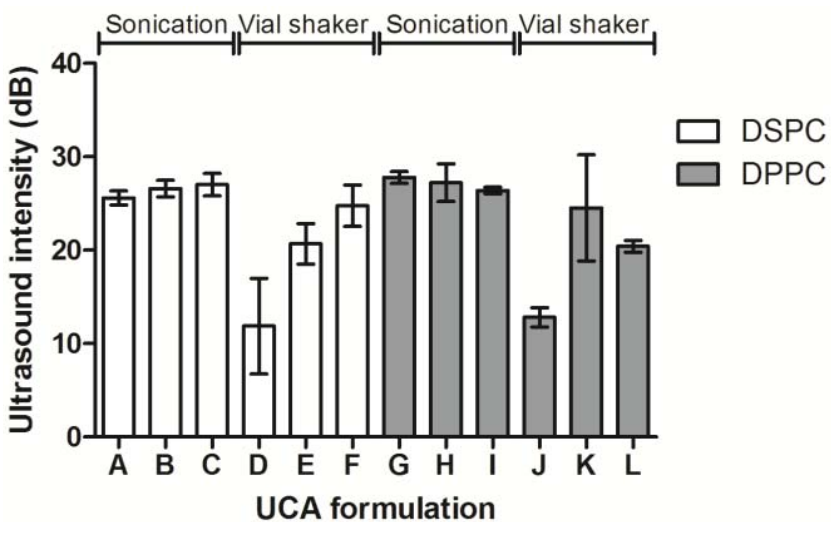

Fig. 3. Ultrasound intensities $(\mathrm{dB})$ at $15 \mathrm{MHz}$ for UCA formulations A-L. Intensities obtained from the PBS control were subtracted from the intensities obtained from the UCA formulations. A-F type UCAs had DSPC as the main coating lipid, while this was DPPC for G-L type UCAs.

microbubbles with larger mean diameters resulted in slightly higher intensities. In addition, UCAs produced by sonication that had similar mean number weighted diameters also showed similar signal intensity in the B-mode scan (A, B, C and G, $\mathrm{H}$, I in Fig. 3). UCA formulation $\mathrm{F}$ had the highest signal of the DSPC-based UCAs made by vial shaking, while this was formulation $\mathrm{K}$ for the DPPC-based UCAs. However, a large $\mathrm{SD}$ was observed for formulation $\mathrm{K}$, due to a nonuniform signal throughout the tube. Two UCA formulations produced by sonication and two produced by vial shaking were selected for further studies. Based on the results so far, UCA formulations $\mathrm{C}$ and I were selected out of the sonication produced UCAs as they contained the highest concentration of microbubbles. For the vial shaking produced UCAs, formulations $\mathrm{F}$ and $\mathrm{L}$ were selected because they gave the highest ultrasound signal in B-mode with the smallest SD.

Images of UCA formulations C, F, I, and L, and TargetReady MicroMarker at high and low concentrations, filtered around their SH frequency (15 MHz), and the corresponding frequency spectra for the three ROIs are presented in Fig. 4. At high UCA concentration, attenuation was dominant for formulations $\mathrm{C}$, I, and $\mathrm{L}$. This attenuation effect is also reflected in the corresponding spectra of these UCAs, where the amplitude of the spectra at the $\mathrm{SH}$ frequency drops about $10 \mathrm{~dB}$ for the deeper ROIs with respect to the highest $\mathrm{SH}$ amplitude. Target-Ready MicroMarker and UCA formulation $\mathrm{F}$ at high concentration had the highest and most homogeneous $\mathrm{SH}$ response throughout the three ROIs. At low UCA concentration, the attenuation effect was less pronounced. The $\mathrm{SH}$ amplitude of Target-Ready MicroMarker dropped about $11 \mathrm{~dB}$ when UCA concentration was reduced by a factor of 20 . The SH amplitude of UCA formulation I at such low concentration was $10 \mathrm{~dB}$ higher than that for Target-Ready MicroMarker and was homogeneous throughout the UCA area.

In all the corresponding spectra of the UCA signals in both high and low concentration, the amplitude of the nonlinear fundamental signal was maximal at the focus of the transducer, where the acoustic energy was at its maximum (ROI 2). At high concentrations, Target-Ready MicroMarker showed 


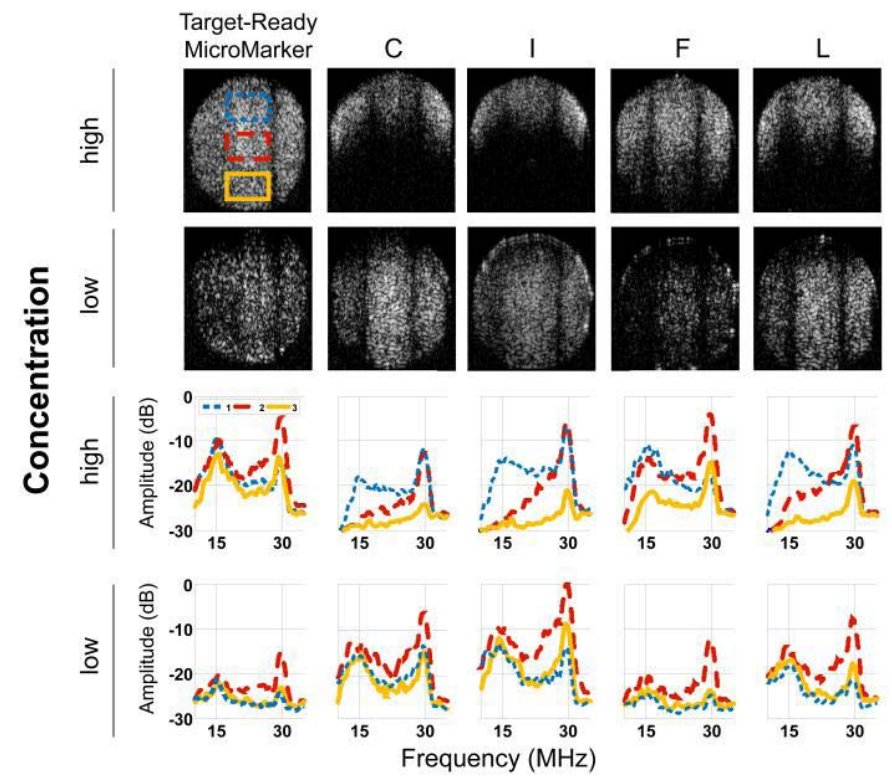

Fig. 4. In vitro $\mathrm{SH}$ images of Target-Ready MicroMarker and formulation $\mathrm{C}, \mathrm{I}, \mathrm{F}$, and $\mathrm{L}$ at high $\left(8.0 \times 10^{6}\right.$ microbubbles $\left./ \mathrm{mL}\right)$ and low $\left(4.0 \times 10^{5}\right.$ microbubbles $\left./ \mathrm{mL}\right)$ concentrations (top) and the corresponding spectra of each ROI at three depths for each image (bottom).

the highest nonlinear response at the fundamental frequency. However, at low concentration, all our in-house produced UCAs had higher nonlinear responses at the fundamental frequency than Target-Ready MicroMarker. The attenuation effect was similar for the SH response and the nonlinear fundamental response. UCA formulations $\mathrm{C}$, I, and L showed higher attenuation than Target-Ready MicroMarker and UCA formulation $\mathrm{F}$.

\section{In Vivo hfCEUS}

Of the four formulations that were studied in vitro for their $\mathrm{SH}$ and nonlinear fundamental response, the two best performing UCA formulations were selected for in vivo hfCEUS studies: $\mathrm{F}$ and $\mathrm{L}$. This decision was based on the fact that both the acoustic signal and microbubble size distribution resembled Target-Ready MicroMarker the closest. Fig. 5 shows the result of the comparison between Target-Ready MicroMarker and UCA formulations $\mathrm{F}$ and $\mathrm{L}$ in the same animal $(n=1)$.

For each recording, three ROIs were drawn: in the focal region (blue), in the middle of the field of view (red), and the bottom of the field of view (yellow). The mean \pm SD intensity in each ROI is shown in Fig. 5 (bottom) for all three UCAs. hfCEUS images revealed slightly lower overall intensities for UCA formulation $\mathrm{F}$ than for Target-Ready MicroMarker. Formulation $\mathrm{L}$ on the other hand revealed only two larger vessels in ROI3, while hardly any intensity increase was observed outside these vessels or in ROI1 and ROI2. Because contrast enhancement of UCA formulation L was negligible, only Target-Ready MicroMarker and UCA formulation F were further evaluated. The TICs corresponding to the example in Fig. 5 for Target-Ready MicroMarker (gray) and UCA formulation $\mathrm{F}$ (black) show very similar behavior between both UCAs in all three ROIs (Fig. 6).
Target-Ready MicroMarker

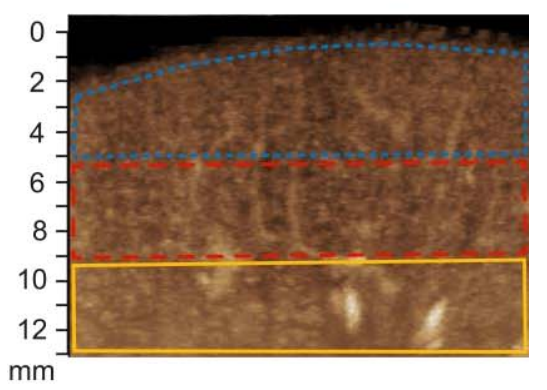

UCA formulation $\mathrm{F}$

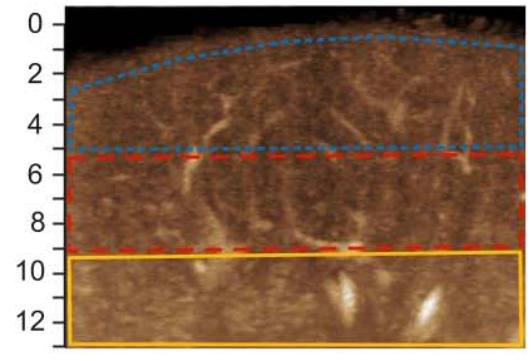

UCA formulation $\mathrm{L}$
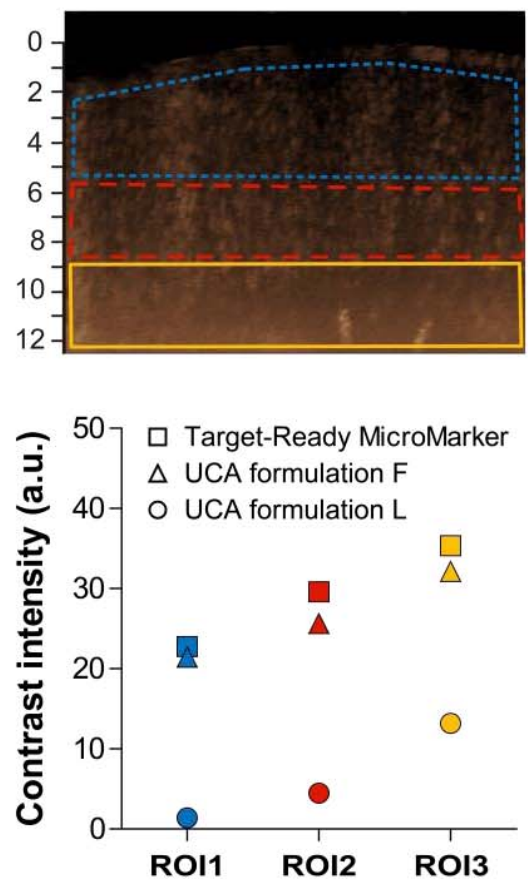

Fig. 5. Examples of in vivo hfCEUS measurements of Target-Ready MicroMarker and formulations $\mathrm{F}$ and $\mathrm{L}$ in the kidney of the same animal. The maximum intensity projection is shown. The bottom graph shows the quantification of intensities in the three different ROIs of which the colors correspond to those in the maximum intensity projections.

The experiments comparing Target-Ready MicroMarker and formulation $\mathrm{F}$ ( $n=7$ in total) confirmed the observation that the total contrast enhancement of Target-Ready MicroMarker was higher, although the difference was lower in ROI3 (Fig. 7). 


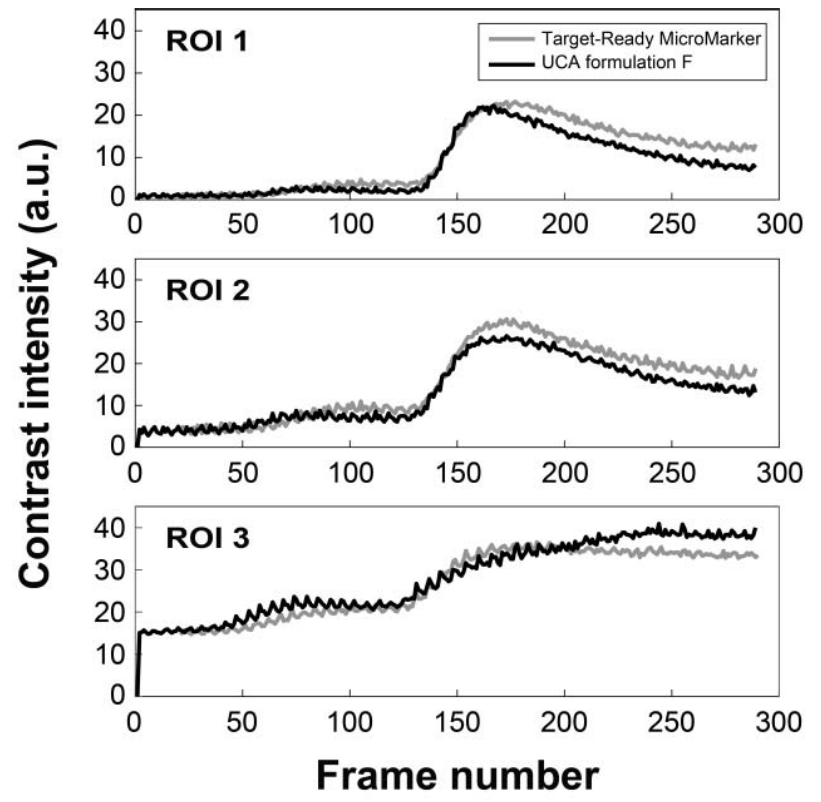

Fig. 6. TICs of Target-Ready MicroMarker (gray) and UCA formulation F (black) for the different ROIs. The TICs correspond to the same injections as the example shown in Fig. 5.

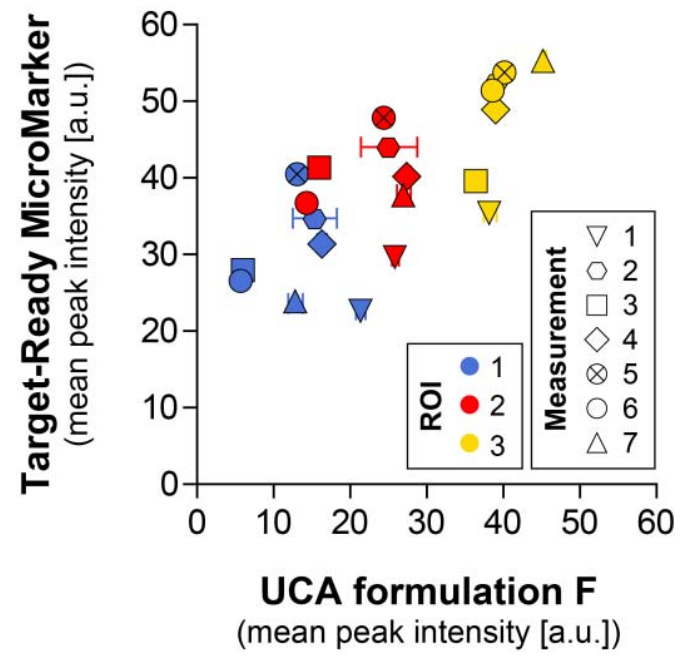

Fig. 7. Complete comparison of Target-Ready MicroMarker and UCA formulation F. The markers indicate the mean peak intensity of each measurement, and the bars indicate the corresponding $\mathrm{SD}(n=7)$. The symbols indicate the data obtained from the same paired injection, whereas $\nabla$ indicates the example shown in Fig. 5 (measurement 1 , animal a). Measurement 2 was done in animal $\mathrm{b}$ and measurement 3-7 in animal $\mathrm{c}$.

This implies lower attenuation for UCA formulation $F$ than for Target-Ready MicroMarker. Since UCA formulation F seemed to better visualize the larger vessels and TargetReady MicroMarker resulted in more enhancement in the microvasculature (see Fig. 5), we quantified the ratio between enhancement in the arteries and the microvasculature for all injections. The symbols that are used in Fig. 7 correspond to those in Fig. 8, and quantification of the example in Fig. $5(\nabla)$ shows better discrimination of the artery from the microvasculature for UCA formulation F. Although this example showed a clear difference, overall the differences

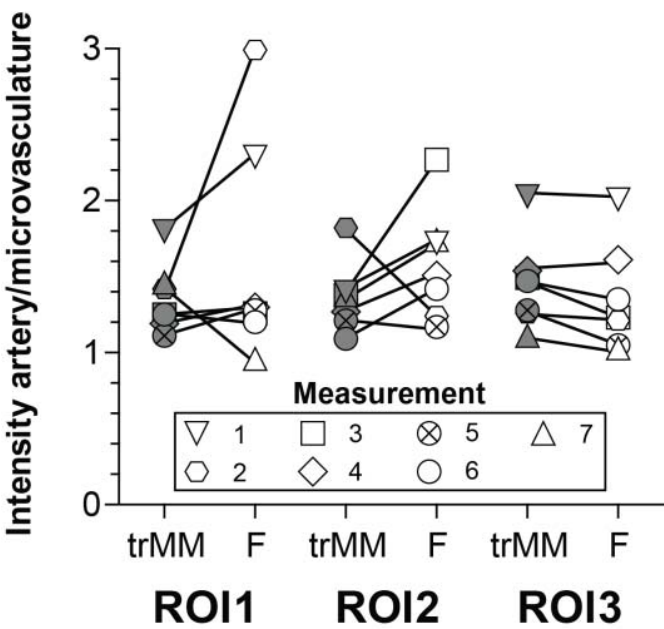

Fig. 8. Comparison of the ratios of contrast enhancement in the arteries and the microvasculature between Target-Ready MicroMarker (gray) and UCA formulation $\mathrm{F}$ (white). The symbols, identical to the symbols in Fig. 7, indicate the data obtained from the same paired injection, whereas $\nabla$ indicates the example shown in Fig. 5 (measurement 1, animal a). Measurement 2 was done in animal $\mathrm{b}$ and measurement 3-7 in animal c. The lines connect the paired injections (injected in random order). Differences between the two UCAs were not significant.

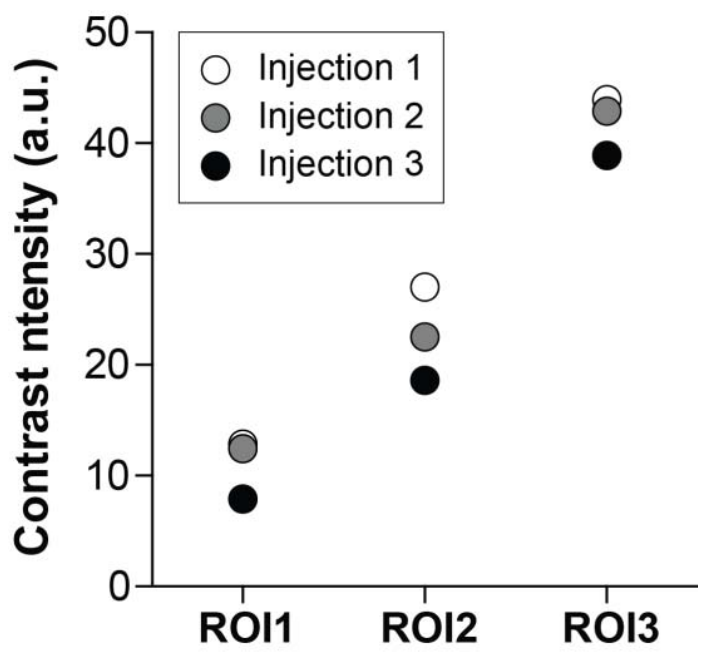

Fig. 9. Repeated injections $(n=3)$ of UCA formulation $\mathrm{F}$ in the same animal. For each injection, contrast enhancement decreased.

between Target-Ready MicroMarker and UCA formulation F were not significant $(p>0.05)$.

We also verified the reproducibility of UCA formulation $\mathrm{F}$ by repetitive injection in the same animal $(n=3)$ and found similar intensities in ROI1 and ROI3 between the first and second injection (Fig. 9). In ROI2, the second injection was $17 \%$ lower than the first injection. The third injection was 39\% lower than the first injection in ROI1, 31\% lower in ROI2, and only $11 \%$ lower in ROI3. We verified that the baseline values before the start of each measurement were comparable. Reproducibility for Target-Ready MicroMarker was not studied. 


\section{DISCUSSION}

In this paper, we showed that one of our in-house produced UCAs for hfCEUS resembled the performance of the commercially available Target-Ready MicroMarker both in vitro and in vivo. The best performing UCA was produced by $45 \mathrm{~s}$ vial shaking and consisted of $92.4 \%$ DSPC and 7.6\% DSPEPEG2000, in an aqueous solution of $70 \%$ PBS, $10 \%$ glycerol, and $20 \%$ propylene glycol with a $\mathrm{C}_{4} \mathrm{~F}_{10}$ gas core.

\section{A. Ultrasound Contrast Agent Preparation}

In our study, microbubble diameters were larger for sonication produced UCAs than when microbubbles were produced by vial shaking, which is in line with what Sirsi et al. [33] found for their in-house produced UCAs with a coating of DSPC and PEG-40 stearate (9:1 molar ratio) and $\mathrm{C}_{4} \mathrm{~F}_{10}$ gas core. In contrast, Moran et al. [34] reported similar number weighted mean diameters of $\sim 0.5 \mu \mathrm{m}$ for their in-house produced UCAs by sonication and vial shaking. Their nitrogen-filled microbubbles had a coating of dipalmitoyl phosphatidylethanolamine (DPPE), phosphatidylcholine, dipalmitoyl phosphatidyl-DL-glycerol, and cholesterol. The contrasting findings in microbubble diameters between the two production methods, i.e., sonication and vial shaking, suggest that the microbubble composition and gas core may also play a role in the size of the produced microbubbles. On the other hand, different probe-sonication devices were used in these studies (Sonicator Ultrasonic Processor XL2020 in our study; Branson Ultrasonics Model $250 \mathrm{~A}$ in [33] and [41]; and Soniprep 150 in [34]), which could have given different ultrasonic power outputs thereby influencing the microbubble size distribution [28].

Kooiman et al. [29] reported that 10-s sonication for the same UCA formulations as A-C (DSPC-based) and G-I (DPPC-based) resulted in microbubbles with a mean number weighted diameter of 4.2 and $3.9 \mu \mathrm{m}$, respectively. Although sonication for $60 \mathrm{~s}$ resulted in smaller microbubbles $(\sim 2 \mu \mathrm{m}$ in mean number weighted diameter; see Table II), the number weighted mean diameter was similar after 60-, 90-, or 120-s sonication. This was also observed by Moran et al. [34] who reported that the number weighted mean diameter of $\sim 0.5 \mu \mathrm{m}$ did not change when the sonication time was varied among 30 , 60,90 , or $300 \mathrm{~s}$ for their lipid-coated microbubbles (coating composition see above). However, the number weighted size distribution of our UCAs produced by sonication had two peaks (0.4-0.5 and $23 \mu \mathrm{m}$; Fig. 2). A longer sonication time seemed to produce lower amounts of large microbubbles $(>2 \mu \mathrm{m})$ and more microbubbles in between the peaks $(0.5-2 \mu \mathrm{m})$. This result is confirmed with the changes in the volume weighted mean diameters, which decreased with increasing sonication time. In addition, microbubble concentrations increased for longer sonication times. It is likely that more microbubbles can be produced during longer sonication times because excess unincorporated lipids are always present when microbubbles are made by sonication [42]. The increase in the concentration and the changes in the size distribution of the UCA suggest that longer sonication times can break up bigger microbubbles into smaller ones.
Our in-house produced UCA formulations D and $\mathbf{J}$ were made by vial shaking in the absence of glycerol and propylene glycol, just as Sirsi et al. [33] and Moran et al. [34] did. On the other hand, glycerol and propylene glycol are often added to increase the fluid viscosity when making UCAs inhouse by vial shaking [30], [35]-[37] and are also present in the clinically approved Definity [21]. When the concentrations of glycerol and propylene glycol were increased from $0 \% / 0 \%$ to $5 \% / 5 \%$ and $10 \% / 20 \% \mathrm{v} / \mathrm{v}$, we found varying results on microbubble size and concentration. For the DPPC-based UCAs (formulations $\mathrm{J}, \mathrm{K}$, and L), increasing the concentration of glycerol and propylene glycol increased the microbubble concentration and the number weighted mean diameter. For the DSPC-based UCAs (formulations D, E, and F), the number weighted mean diameter followed the same trend. The concentration of the microbubbles, on the other hand, first increased with 5\%/5\% glycerol and propylene glycol and decreased for the highest concentration. In addition, UCA formulations $\mathrm{F}$ and $\mathrm{L}$ were more stable after $2 \mathrm{~h}$ than those without glycerol and propylene glycol. This suggests that glycerol and propylene glycol can play a role not only in increasing the concentration of the microbubbles, but also on the stability of the produced microbubbles. This increased stability of UCA formulations $\mathrm{F}$ and $\mathrm{L}$ was also observed in the increased intensity in the in vitro B-mode images of these UCAs.

\section{B. In Vitro hfCEUS}

In an in vitro setup, mimicking a practical imaging condition, we showed that both the shell microstructure of microbubbles as well as their size distribution have a considerable impact on their nonlinear behavior both at the $\mathrm{SH}$ and fundamental frequencies. All our homemade UCAs showed high nonlinear behavior when excited at $30 \mathrm{MHz}$, which was comparable with the commercially available Target-Ready MicroMarker. Such high nonlinear response at both the SH and fundamental frequencies can be attributed to the small sizes of all studied microbubbles. The larger microbubbles in formulations $\mathrm{C}$ and I (2-3 $\mu \mathrm{m})$ accounted for more attenuation at high concentrations. This hypothesis is confirmed by the lower attenuation of the smaller microbubbles in UCAs F and L, while the nonlinear responses remained high. The attenuation effect was more pronounced for the SH component than the nonlinear responses at the fundamental frequency. This is perhaps due to the threshold behavior of SH oscillations [14]. The microbubbles at the lower part of the focal zone may not have been excited with sufficiently high pressures to undergo $\mathrm{SH}$ oscillation. However, when the concentration was $20 \times$ lower, the $\mathrm{SH}$ response of these larger microbubbles became stronger and more homogeneous throughout the sample.

Comparison of UCA formulations $\mathrm{C}$ and I with similar size distributions but different main lipid component showed that the DPPC-based UCA formulation I showed higher nonlinear responses at both the fundamental and $\mathrm{SH}$ frequencies. At low concentration, the response of UCA formulation I at the fundamental and SH frequency was 6 and $3 \mathrm{~dB}$ higher, respectively, than for the DSPC-based UCA formulation C. This higher SH response has also been reported in [20] utilizing optical single microbubble spectroscopy in the range of $1-4 \mathrm{MHz}$. 
The small size of Target-Ready MicroMarker microbubbles seems to play an important role in its nonlinear behavior, particularly for the SH response at high-frequency excitation. The majority of the microbubbles have a diameter below $1.5 \mu \mathrm{m}$, corresponding to a resonance frequency around and higher than $15 \mathrm{MHz}$ [43], [44]. It is conventionally thought that SH generation is achieved most readily (i.e., at lowest pressure threshold) when microbubbles are excited at twice their resonance frequency [16]. This means that for the majority of the Target-Ready MicroMarker microbubbles, the $30-\mathrm{MHz}$ excitation used in this paper is around twice their resonance frequency. Indeed, high amplitude $\mathrm{SH}$ response of TargetReady MicroMarker UCA has previously been reported by Helfield et al. [19], although they studied individual microbubbles in an acoustical setup. Another reason for the high $\mathrm{SH}$ behavior of the UCAs we observed may be the rectangular shape of the excitation used in the Vevo2100 scanner. It has been reported that rectangular-shaped excitations generate a self-demodulation signal, which enhances the SH response of microbubbles [14], [15]. Our in vitro results show that TargetReady MicroMarker is also producing a strong nonlinear response at the fundamental frequency. This characteristic is already being utilized in the Vevo2100 scanner as the default nonlinear contrast mode with amplitude modulation pulse sequence to improve the contrast to tissue ratio in hfCEUS.

It has been shown before that smaller microbubbles provide higher contrast for hfCEUS imaging. Goertz et al. [45] reported that the decantation technique to isolate smaller Definity microbubbles can be employed to produce an attenuation pattern that appears more favorable for hfCEUS. Also Moran et al. [34] have shown that decreasing the mean diameter of the lipid-based sonication UCAs significantly increased the mean backscattering power at $40 \mathrm{MHz}$. Peyman et al. [46] showed that the majority of the signal measured using the VisualSonics Vevo770 (40 MHz) in the mixed microbubble/nanobubble population was attributable to the nanobubbles, with a much lower proportion of the signal coming from microbubbles. Our results are in agreement with these studies.

We showed that at $30-\mathrm{MHz}$ transmit frequency and at low UCA concentration, DPPC-based UCAs behaved more nonlinearly than those based on DSPC. At higher concentration, smaller microbubbles (Target-Ready MicroMarker and UCA formulation F) produced the most homogeneous $\mathrm{SH}$ responses. For UCA formulations C and I, Kooiman et al. [29] observed differences in lipid distribution. The DPPC lipid has a lower elastic compressibility modulus than DSPC [47], [48], which may be related to $\mathrm{SH}$ behavior as our DPPC-based UCAs showed more SH behavior than our DSPC-based UCAs, as reported before for single microbubbles [20]. Unfortunately, the coating composition of Target-Ready MicroMarker is unknown, so we do not know how our homemade UCAs resemble the composition of Target-Ready MicroMarker.

\section{In Vivo hfCEUS}

We studied UCA formulations $\mathrm{F}$ and $\mathrm{L}$ in vivo and found that the overall performance of UCA formulation F was similar to that of Target-Ready MicroMarker. UCA formulation L, on the other hand, resulted in hardly any contrast enhancement despite the fact that both in-house produced UCAs had similar number-weighted mean diameters and the concentration of microbubbles was even higher for UCA formulation L. The most important difference between the two UCAs is their composition and the associated differences between DSPC as main component (formulation F) and DPPC (formulation L) in terms of microstructure [29, [49]-[51] and acoustic behavior in vitro [20]. Using an Acuson Sequoia 512 in nonlinear imaging mode at $7 \mathrm{MHz}$ (15L8 transducer), the in vitro halflife of DPPC-based UCAs (DPPC:DSPE-PEG5000, 9:1, inhouse produced by vial shaking) was $10 \times$ lower than for those based on DSPC, whereas the half-life was better in vivo, but still $5 \times$ lower than for DSPC-based UCAs [52]. Although these and our in-house produced DPPC-based UCAs did not perform well in vivo, the commercially available and clinically approved UCA Definity that is also DPPC-based [21] has been shown to provide good contrast enhancement in pigs [53] and humans [54], [55]. It has to be noted that the other components of Definity are DPPA and DPPEmPEG5000 [21], which can alter the acoustic properties and stability.

Other research groups have also characterized their own inhouse produced UCAs for hfCEUS, but either characterized them acoustically in vitro [19], [20], [46], [56]-[58] and/or imaged them in vivo [33], [46], instead of imaging them in both situations. Although acoustic characterization of single microbubbles provides insights in microbubble behavior, imaging of microbubbles in vitro in a controlled and simplified setup is essential before translation toward complex in vivo applications. Neither in vivo studies compared their in-house produced UCAs to a commercially available UCA as a reference. Peyman et al. [46] imaged their microbubbles (DPPC:DSPE-PEG2000, 4:1, microfluidic production) in the mouse aorta at $40 \mathrm{MHz}$ in fundamental mode using the Vevo770, which resulted in hardly any contrast enhancement. Simultaneously, with their microbubbles, they also produced nanobubbles ( 200 -nm diameter), which provided $3 \times$ higher contrast peak intensities. Sirsi et al. [33] isolated microbubbles (DSPC:PEG40-stearate, 9:1 molar ratio, both sonication and vial shaking) of distinct size populations (1-2, 2-4, and 6-8 $\mu \mathrm{m}$ ) and determined their acoustic impact directly in vivo in the mouse kidney using 40-MHz fundamental mode imaging using the Vevo770. Surprisingly, they report that the smaller microbubbles $(1-2 \mu \mathrm{m})$ resulted in higher attenuation and less echogenicity than the larger microbubbles $(6-8 \mu \mathrm{m})$. One reason for these contradictory results could be the differences in microbubble shell composition in these studies. Also, in the study conducted by Sirsi et al. [33], the targets were the small capillaries in the mouse kidney, while Peyman et al. [46] quantified the UCA contrast in the mouse aorta that is a much larger vessel. Both the concentration of the microbubbles in the ROI and the differences in ambient pressures in kidney capillaries and the aorta could have played a role in these contradictory findings.

The TICs of UCA formulation $F$ and Target-Ready MicroMarker were very similar, but slightly higher peaks 
and longer contrast persistence were found for Target-Ready MicroMarker. The prolonged contrast enhancement is likely due to the higher acoustic stability of Target-Ready MicroMarker. These microbubbles not only contain a lipid shell, but are also covered by streptavidin, which has been shown to increase the stiffness and therefore reduce acoustic dissolution in vitro [24]. The higher contrast peak intensity for TargetReady MicroMarker was shown to be present for all injections, but the difference with UCA formulation F was smaller in the deeper cortex. This may result from the higher concentration of formulation F microbubbles that were injected and appeared in the large vessels in the deeper cortex. The differences in concentration between Target-Ready MicroMarker and UCA formulation $\mathrm{F}$ influenced the interpretation of our results. We concluded that the performance between both UCAs was comparable, but it has to be taken into account that an injection of $1 \mathrm{~mL}$ UCA formulation $F$ contained $\sim 7 \times$ more microbubbles than $1 \mathrm{~mL}$ of Target-Ready MicroMarker. However, both concentrations are the same order of magnitude as the commercially available ones, since a typical human dose of Definity contains $4.2 \times 10^{9}$ microbubbles [21] and a typical dose of SonoVue/Lumason varies between $3.0 \times 10^{8}$ and $1.1 \times 10^{9}$ microbubbles [27]. In addition, the concentration of microbubbles between batches of Target-Ready MicroMarker that were used in our experiments varied between $2.6 \times 10^{8}$ and $1.3 \times 10^{9}$ microbubbles $/ \mathrm{mL}(n=5)$. These concentrations were assessed by us and are in line with what others have reported, namely, $8.4 \times 10^{8}$ [25], $9.2 \times 10^{8}$ [59], and $1.9 \times 10^{9}$ [60]. The batch to batch variability in microbubble concentration for our UCA formulation $\mathrm{F}$ ranged from $2.1 \times 10^{9}$ to $5.5 \times 10^{9}$ microbubbles $/ \mathrm{mL}$ ( $n=5$ batches) and was therefore narrower than for Target-Ready MicroMarker. The variation in the mean microbubble diameter (both number and volume weighted) was similar for Target-Ready MicroMarker and our in-house produced UCA formulation F. The number weighted mean diameter for Target-Ready MicroMarker was $1.8 \pm 0.4$ and the volume-weighted mean diameter was $4.6 \pm 0.8$, while this was $1.2 \pm 0.1$ and $5.6 \pm 1.0$ for our in-house produced F type UCA (both $n=$ 5 batches). The variability in microbubble size and concentration can be caused by vial handling, even within manufacturer's recommended procedures, as has been suggested by Goertz et al. [45].

Repeated bolus injections of UCA formulation $\mathrm{F}$ in the same animal resulted in significantly less enhancement for the third injection. Others have reported differences between consecutive injections in cats [61] and mice [62], [63]. In the kidney and spleen of healthy cats, the second bolus injection of SonoVue resulted in higher peak intensities [61]. Dizeux et al. [63] reported that the maximum intensity (peak enhancement) was constant for four consecutive injections in the healthy renal murine cortex, but increased from the second to the fourth injection in renal tumor tissue. In contrast, Rix et al. [62] reported constant peak enhancement in murine liver tumors and decreasing peak enhancement with consecutive injections in the healthy liver. We can only conclude that variability in consecutive injections is a known issue for CEUS and differs between organisms and organs.

\section{Limitations}

The main limitation of using in-house produced UCAs is that it can be challenging to produce them under good manufacturing practice conditions [64]. For example, batch to batch reproducibility may be an issue, as is sterility. However, for terminal preclinical experiments as in our study, the UCAs do not have to be sterile. For longitudinal preclinical experiments, there are options for sterilization of lipid formulations [65], [66]. In our in vivo experiments, we used nonlinear fundamental imaging at $18 \mathrm{MHz}$ with amplitude modulation instead of SH imaging with pulse inversion at $30 \mathrm{MHz}$ as used in vitro. Although SH imaging is free from nonlinear propagation artifacts, nonlinear fundamental imaging with amplitude modulation provides higher contrast to tissue ratio with Vevo2100 [15].

\section{CONClusion}

Our results suggest that our UCA formulation F performs equally well as Target-Ready MicroMarker in hfCEUS imaging. This paper shows that small UCAs having high nonlinear responses for hfCEUS can be produced by mechanical agitation, a shell composition of 92.4\% DSPC, 6.4\% DSPE-PEG2000, and 1.2\% DSPE-PEG2000-biotin encapsulating a $\mathrm{C}_{4} \mathrm{~F}_{10}$ gas core, in a PBS-based liquid with $10 \%$ glycerol and $20 \%$ propylene glycol.

\section{ACKNOWLEDGMENT}

The authors would like to thank all colleagues who made the experiments possible for their help and support, especially Y. Ince from the Department of Translational Physiology, Academic Medical Center, Amsterdam, the Netherlands, and the Department of Intensive Care Adults, Erasmus MC, Rotterdam, the Netherlands; and M. Sorelli from the Department of Intensive Care Adults, Erasmus MC, Rotterdam, the Netherlands, and the Department of Information Engineering, University of Florence, Florence, Italy.

\section{REFERENCES}

[1] F. S. Foster, J. Hossack, and S. L. Adamson, "Micro-ultrasound for preclinical imaging," Interface Focus, vol. 1, no. 4, pp. 576-601, 2011.

[2] F. M. Reid, N. A. Niemuth, S. M. Shumaker, J. D. Waugh, and J. S. Graham, "Biomechanical monitoring of cutaneous sulfur mustardinduced lesions in the weanling pig model for depth of injury," Skin Res. Technol., vol. 13, pp. 217-225, May 2007.

[3] X. Yan, M. Li, Z. Chen, Y. Zhu, Y. Song, and H. Zhang, "Schlemm's canal and trabecular meshwork in eyes with primary open angle glaucoma: A comparative study using high-frequency ultrasound biomicroscopy," PLOS ONE, vol. 11, p. e0145824, Jan. 2016.

[4] D. L. Jovanovic and Z. U. Pesic, "Preoperative skin tumours thickness determination by high-frequency ultrasound on head and neck region," J. Eur. Acad. Dermatol Venereol, vol. 27, pp. 251-253, Feb. 2013.

[5] S. Ghai et al., "Assessing cancer risk on novel $29 \mathrm{MHz}$ micro-ultrasound images of the prostate: Creation of the micro-ultrasound protocol for prostate risk identification," J. Urol., vol. 196, pp. 562-569, Aug. 2016.

[6] C. L. de Korte, H. H. Hansen, and A. F. van der Steen, "Vascular ultrasound for atherosclerosis imaging," Interface Focus, vol. 1, pp. 565-575, Aug. 2011.

[7] V. Cantisani et al., "Ambrosio, "Growing indications for CEUS: The kidney, testis, lymph nodes, thyroid, prostate, and small bowel," Eur. J. Radiol., vol. 84, pp. 1675-1684, Sep. 2015.

[8] A. Alzaraa et al., "Contrast-enhanced ultrasound in the preoperative, intraoperative and postoperative assessment of liver lesions," Hepatol. Res., vol. 43, pp. 809-819, Aug. 2013. 
[9] A. L. Klibanov, "Ultrasound contrast materials in cardiovascular medicine: From perfusion assessment to molecular imaging," J. Cardiovascular Transl. Res., vol. 6, no. 5, pp. 729-739, 2013

[10] K. Kooiman, H. J. Vos, M. Versluis, and N. de Jong, "Acoustic behavior of microbubbles and implications for drug delivery," Adv. Drug Del. Rev., vol. 72C, pp. 28-48, Jun. 2014.

[11] D. E. Goertz et al., "High frequency nonlinear B-scan imaging of microbubble contrast agents," IEEE Trans. Ultrason., Ferroelect., Freq. Control, vol. 52, no. 1, pp. 65-79, Jan. 2005.

[12] A. Needles et al., "Nonlinear contrast imaging with an arraybased micro-ultrasound system," Ultrasound Med. Biol., vol. 36, pp. 2097-2106, Dec. 2010.

[13] G. L. ten Kate et al., "Far-wall pseudoenhancement during contrastenhanced ultrasound of the carotid arteries: Clinical description and in vitro reproduction," Ultrasound Med. Biol., vol. 38, no. 4, pp. 593-600, 2012.

[14] V. Daeichin, T. Faez, G. Renaud, J. G. Bosch, A. F. van der Steen, and N. de Jong, "Effect of self-demodulation on the subharmonic response of contrast agent microbubbles," Phys. Med. Biol., vol. 57, no. 12, pp. 3675-3691, 2012

[15] V. Daeichin, J. G. Bosch, A. Needles, F. S. Foster, A. van der Steen, and N. de Jong, "Subharmonic, non-linear fundamental and ultraharmonic imaging of microbubble contrast at high frequencies," Ultrasound Med. Biol., vol. 41, no. 2, pp. 486-497, 2015.

[16] A. Katiyar and K. Sarkar, "Excitation threshold for subharmonic generation from contrast microbubbles," J. Acoust. Soc. Amer., vol. 130, no. 5, pp. 3137-3147, 2011

[17] M. R. Sprague, E. Chérin, D. E. Goertz, and F. S. Foster, "Nonlinear emission from individual bound microbubbles at high frequencies," Ultrasound Med. Biol., vol. 36, pp. 313-324, Feb. 2010.

[18] T. G. Leighton, The Acoustic Bubble. London, U.K.: Academic, 1994.

[19] B. L. Helfield, E. Cherin, F. S. Foster, and D. E. Goertz, "Investigating the subharmonic response of individual phospholipid encapsulated microbubbles at high frequencies: A comparative study of five agents,' Ultrasound Med. Biol., vol. 38, pp. 846-863, May 2012.

[20] T. van Rooij et al., "Non-linear response and viscoelastic properties of lipid-coated microbubbles: DSPC versus DPPC,' Ultrasound Med. Biol., vol. 41, pp. 1432-1445, May 2015.

[21] Definity Safety Label FDA/Center for Drug Evaluation and Research, L. M. Imaging, Silver Spring, MD, USA, Oct. 2011

[22] Vevo Micromarker Non-Targeted Contrast Agent Kit, Vis. Sonics Inc., Toronto, ON, Canada, 2016.

[23] J. J. Rychak et al., "Microultrasound molecular imaging of vascular endothelial growth factor receptor 2 in a mouse model of tumor angiogenesis," Molecular Imag., vol. 6, pp. 289-296, Sep./Oct. 2007.

[24] T. van Rooij, V. Daeichin, I. Skachkov, N. de Jong, and K. Kooiman, "Targeted ultrasound contrast agents for ultrasound molecular imaging and therapy," Int. J. Hyperthermia, vol. 31, pp. 106-190, Mar. 2015.

[25] J. K. Willmann et al., "US imaging of tumor angiogenesis with microbubbles targeted to vascular endothelial growth factor receptor type 2 in mice," Radiology, vol. 246, pp. 508-518, Feb. 2008.

[26] V. Daeichin, K. Kooiman, I. Skachkov, J. G. Bosch, A. F. van der Steen, and N. de Jong, "Optimization of ultrasound contrast agent for high frequency ultrasound molecular imaging using subharmonic oscillation," in Proc. IEEE Ultrason. Symp., Chicago, IL, USA, Sep. 2014, pp. 1766-1769.

[27] Lumason Safety Label FDA/Center for Drug Evaluation and Research, B. D. Inc, Silver Spring, MD, USA, Mar. 20162016.

[28] E. Stride and M. Edirisinghe, "Novel microbubble preparation technologies," Soft Matter, vol. 4, no. 12, pp. 2350-2359, 2008.

[29] K. Kooiman et al., "DSPC or DPPC as main shell component influences ligand distribution and binding area of lipid-coated targeted microbubbles,” Eur. J. Lipid Sci. Technol., vol. 116, no. 9, pp. 1217-1227, 2014

[30] B. Geers, I. Lentacker, N. N. Sanders, J. Demeester, S. Meairs, and S. C. De Smedt, "Self-assembled liposome-loaded microbubbles: The missing link for safe and efficient ultrasound triggered drug-delivery," J. Control Release, vol. 152, pp. 249-256, Jun. 2011.

[31] T. Segers, L. de Rond, N. de Jong, M. Borden, and M. Versluis, "Stability of monodisperse phospholipid-coated microbubbles formed by flowfocusing at high production rates," Langmuir, vol. 32, pp. 3937-3944, Apr. 2016.

[32] A. L. Klibanov et al., "Detection of individual microbubbles of ultrasound contrast agents: Imaging of free-floating and targeted bubbles," Invest. Radiol., vol. 39, pp. 187-195, Mar. 2004.

[33] S. Sirsi, J. Feshitan, J. Kwan, S. Homma, and M. Borden, "Effect of microbubble size on fundamental mode high frequency ultrasound imaging in mice," Ultrasound Med. Biol., vol. 36, pp. 935-948, Jun. 2010.
[34] C. M. Moran et al., "Manufacture and acoustical characterisation of a high-frequency contrast agent for targeting applications," Ultrasound Med. Biol., vol. 32, pp. 421-428, Mar. 2006.

[35] M. A. Borden et al., "Lateral phase separation in lipid-coated microbubbles," Langmuir, vol. 22, pp. 4291-4297, Apr. 2006.

[36] S. Wang, G. Samiotaki, O. Olumolade, J. A. Feshitan, and E. E. Konofagou, "Microbubble type and distribution dependence of focused ultrasound-induced blood-brain barrier opening," Ultrasound Med. Biol., vol. 40, pp. 130-137, Jan. 2014.

[37] M. Kaya, T. S. V. Gregory, and P. A. Dayton, "Changes in lipidencapsulated microbubble population during continuous infusion and methods to maintain consistency," Ultrasound Med. Biol., vol. 35, pp. $1748-1755$, Oct. 2009

[38] C. C. Shen and P. C. Li, "Pulse-inversion-based fundamental imaging for contrast detection," IEEE Trans. Ultrason., Ferroelect., Freq. Control, vol. 50, no. 9, pp. 1124-1133, Sep. 2003.

[39] V. Daeichin et al., "Quantification of bound microbubbles in ultrasound molecular imaging," IEEE Trans. Ultrason., Ferroelect., Freq. Control, vol. 62, no. 6, pp. 1190-1200, Jun. 2015.

[40] A. Hoogi et al., "Quantitative analysis of ultrasound contrast flow behavior in carotid plaque neovasculature," Ultrasound Med. Biol., vol. 38, pp. 2072-2083, Dec. 2012.

[41] J. A. Feshitan, C. C. Chen, J. J. Kwan, and M. A. Borden, "Microbubble size isolation by differential centrifugation," J. Colloid Interface Sci., vol. 329, pp. 316-324, Jan. 2009.

[42] A. L. Klibanov, "Preparation of targeted microbubbles: Ultrasound contrast agents for molecular imaging," Med. Biol. Eng. Comput., vol. 47 , pp. 875-882, Aug. 2009.

[43] M. Minnaert, "XVI. On musical air-bubbles and the sounds of running water," London, Edinburgh, Dublin Phil. Mag. J. Sci., vol. 16, no. 104, pp. 235-248, 1933.

[44] B. L. Helfield, B. Y. C. Leung, X. Huo, and D. E. Goertz, "Scaling of the viscoelastic shell properties of phospholipid encapsulated microbubbles with ultrasound frequency," Ultrasonics, vol. 54, pp. 1419-1424, Aug. 2014.

[45] D. E. Goertz, N. de Jong, and A. F. W. van der Steen, "Attenuation and size distribution measurements of definity and manipulated definity populations," Ultrasound Med. Biol., vol. 33, pp. 1376-1388, Sep. 2007.

[46] S. A. Peyman et al., "On-chip preparation of nanoscale contrast agents towards high-resolution ultrasound imaging," Lab Chip, vol. 16, pp. 679-687, Feb. 2016.

[47] P. Dynarowicz-Łătka and K. Hac-Wydro, "Interactions between phosphatidylcholines and cholesterol in monolayers at the air/water interface," Colloids Surfaces B, Biointerfaces, vol. 37, pp. 5-21, Aug. 2004.

[48] Y. Kurniawan, C. Scholz, and G. D. Bothun, " $n$-butanol partitioning into phase-separated heterogeneous lipid monolayers," Langmuir, vol. 29, no. 34, pp. 10817-10823, Aug. 2013.

[49] M. M. Lozano and L. Longo, "Complex formation and other phase transformations mapped in saturated phosphatidylcholine/DSPE-PEG2000 monolayers," Soft Matter, vol. 5, pp. 1822-1834, Feb. 2009.

[50] V. M. Kaganer, H. Mohwald, and P. Dutta, "Structure and phase transitions in Langmuir monolayers," Rev. Mod. Phys., vol. 71, pp. 779-819, Apr. 1999.

[51] M. A. Borden, G. Pu, G. J. Runner, and M. L. Longo, "Surface phase behavior and microstructure of lipid/PEG-emulsifier monolayer-coated microbubbles," Colloids Surf. B, Biointerfaces, vol. 35, pp. 209-223, Jun. 2004.

[52] S. Garg, A. A. Thomas, and M. A. Borden, "The effect of lipid monolayer in-plane rigidity on in vivo microbubble circulation persistence," Biomaterials, vol. 34, pp. 6862-6870, Sep. 2013.

[53] A. F. Schinkel et al., "Contrast-enhanced ultrasound for imaging vasa vasorum: Comparison with histopathology in a swine model of atherosclerosis," Eur. J. Echocardiogr, vol. 11, pp. 659-664, Sep. 2010.

[54] M. S. Dolan et al., "Safety and efficacy of commercially available ultrasound contrast agents for rest and stress echocardiography a multicenter experience," J. Amer. College Cardiol., vol. 53, pp. 32-38, Jan. 2009.

[55] D. W. Kitzman, M. E. Goldman, L. D. Gillam, J. L. Cohen, G. P. Aurigemma, and J. S. Gottdiener, "Efficacy and safety of the novel ultrasound contrast agent perflutren (definity) in patients with suboptimal baseline left ventricular echocardiographic images?" Amer. J. Cardiol., vol. 86, pp. 669-674, Sep. 2000

[56] M. A. Borden and M. L. Longo, "Dissolution behavior of lipid monolayer-coated, air-filled microbubbles: Effect of lipid hydrophobic chain length," Langmuir, vol. 18, pp. 9225-9233, Nov. 2002.

[57] Y. Luan et al., "Acoustical properties of individual liposome-loaded microbubbles," Ultrasound Med. Biol., vol. 38, pp. 2174-2185, Dec. 2012. 
[58] M. A. Parrales, J. M. Fernandez, M. Perez-Saborid, J. A. Kopechek, and T. M. Porter, "Acoustic characterization of monodisperse lipidcoated microbubbles: Relationship between size and shell viscoelastic properties," J. Acoust. Soc. Amer., vol. 136, pp. 1077-1084, Sep. 2014

[59] A. Lyshchik, A. C. Fleischer, J. Huamani, D. E. Hallahan, M. Brissova, and J. C. Gore, "Molecular imaging of vascular endothelial growth factor receptor 2 expression using targeted contrast-enhanced highfrequency ultrasonography," J. Ultrasound Med., vol. 26, pp. 1575-1586, Nov. 2007.

[60] S. A. Stapleton, A. Needles, E. Henderson, and F. S. Foster, "Concentration requirements for subharmonic quantitative contrast enhanced high frequency ultrasound flow studies," in Proc. IEEE Ultrason. Symp., Oct. 2007, pp. 1061-1064.

[61] E. Stock, K. Vanderperren, H. Haers, L. Duchateau, M. Hesta, and J. H. Saunders, "Quantitative differences between the first and second injection of contrast agent in contrast-enhanced ultrasonography of feline kidneys and spleen," Ultrasound Med. Biol., vol. 43, no. 2, pp. 500-504, Feb. 2017, doi: 10.1016/j.ultrasmedbio.2016.09.013.

[62] A. Rix et al., "Influence of repetitive contrast agent injections on functional and molecular ultrasound measurements," Ultrasound Med. Biol., vol. 40, pp. 2468-2475, Oct. 2014.

[63] A. Dizeux, T. Payen, G. Barrois, D. Le G. Buffello, and S. L. Bridal, "Reproducibility of contrast-enhanced ultrasound in mice with controlled injection," Molecular Imag. Biol., vol. 18, no. 5, pp. 651-658, Apr. 2016.

[64] W. H. Organization, WHO Good Manufacturing Practices for Pharmaceutical Products: Main Principles. Geneva, Switzerland: World Health Organization, 2011.

[65] M.-R. Toh and G. N. C. Chiu, "Liposomes as sterile preparations and limitations of sterilisation techniques in liposomal manufacturing," Asian J. Pharmaceutical Sci., vol. 8, no. 2, pp. 88-95, 2013.

[66] P. K. Hui and W. R. Diluzio, "Stabilization and terminal sterilization of phospholipid formulations," U.S. Patent EP 1420880 A4, Mar. 20, 2002.

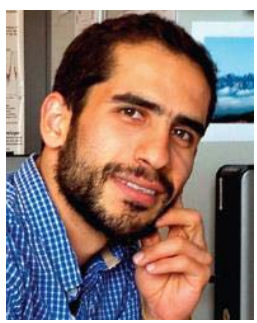

Verya Daeichin (M'12) received the Ph.D. degree in microultrasound molecular imaging from the Erasmus Medical Center, Rotterdam, The Netherlands.

He was a Post-Doctoral Researcher at the Erasmus Medical Centre, where he focused on intravascular photoacoustic imaging for 18 months. Since 2016, he has been a Senior Scientist with the Acoustical Wavefield Imaging Group, Delft University of Technology, Delft, The Netherlands. He is involved in various medical ultrasound projects, focusing on novel ultrasound contrast agent, transducer design, manufacturing, and applications.

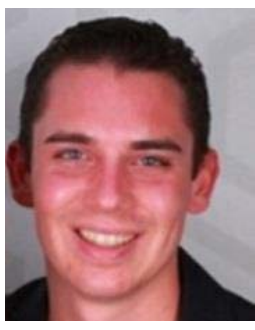

Tom van Rooij (M'12) was born in Eindhoven, The Netherlands, in 1987. He received the B.Sc. degree in biomedical engineering and the M.Sc. degree in medical engineering from the Eindhoven University of Technology, Eindhoven, in 2008 and 2012, respectively. His thesis focuses on contrast agents for ultrasound imaging and therapy by using ultrahigh-speed optical imaging techniques, and in vitro and in vivo ultrasound imaging.

He held a teaching position at the Biomedical NMR Group, Department of Biomedical Engineering, Eindhoven University of Technology. In 2012, he started a Ph.D. project at the Department of Biomedical Engineering, Thorax Center, Erasmus MC, Rotterdam, The Netherlands.

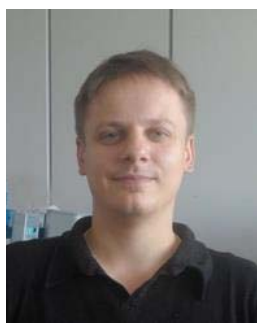

Ilya Skachkov received the M.Sc. degree in cell biology from the Faculty of Biology, Lomonosov Moscow State University, Moscow, Russia, in 2009, and the Ph.D. degree from the Department of Biomedical Engineering, Thoraxcenter, Erasmus MC, Rotterdam, The Netherlands, in 2016.

From 2009 to 2010, he was with the Institute for Biomedical Problems, Moscow, where he focused on molecular pathways involved in muscle degeneration in response to hypogravity. In 2016, he joined the Group of Prof. C.T.W. Moonen, University Medical Center Utrecht, Utrecht, The Netherlands, as a Post-Doctoral Researcher, where he focused on ultrasound and microbubble mediated drug delivery. His current research interests include ultrasound contrast agents for molecular imaging and drug delivery, and unraveling mechanisms of sonoporation.

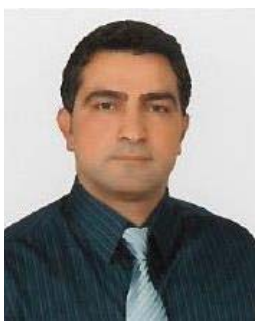

Bulent Ergin was born in Istanbul, Turkey, in 1978. He received the bachelor's degree in biology, the master's degree, and the Ph.D. degree from the Zoology Division, University of Istanbul, Istanbul, in 2004, 2008, and 2013, respectively.

Since 2011, he has been a Researcher with the Department of Translational Physiology, Academic Medical Center, University of Amsterdam, Amsterdam, The Netherlands. He is currently a Physiologist, experienced in small or large animal surgery, anesthesiology, histology, electron microscopy, immunohistochemistry, and biochemical analysis in a model of sepsis, hemorrhagic shock, hemodilution, ischemia/reperfusion, diabetes, and liver failure.

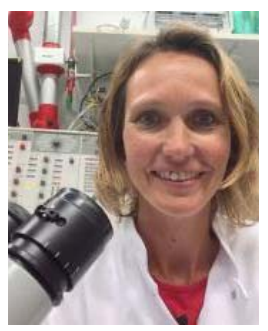

Patricia A. C. Specht received the bachelor of applied science degree from the Department of Anesthesiology, Erasmus MC, Rotterdam, The Netherlands, and the bachelor's degree with a specialization in biology and medical laboratory research from the Department of Anesthesiology, Rotterdam University of Applied Sciences, Rotterdam, in 1999

In 2000, she joined the Department of Physiology, VU University Medical Center, Amsterdam, The Netherlands. In the same year, she joined the Department of Pediatric Surgery. In 2006, she joined the Department of Anesthesiology, Erasmus MC, where she specialized in surgery, anesthesia, and ventilation in animal research models.

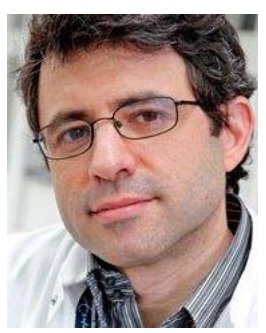

Alexandre Lima is currently a Senior Post-Doctoral Researcher with Erasmus MC, Rotterdam, The Netherlands. He has expertise in planning and conducting clinical research through scientific evidencebased data to monitor research methodologies and clinical/experimental studies design.

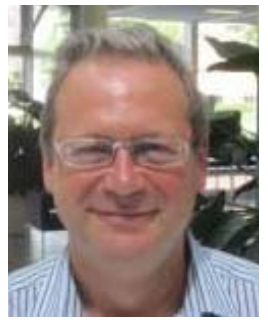

Can Ince received the B.Sc. degree in electrical and electronic engineering from the University of Birmingham, Birmingham, U.K., the M.Sc. degree in electrical and electronic engineering from the Technical University of Delft, Delft, The Netherlands, and the Ph.D. degree in immunology/physiology from the Department of Infectious Diseases, University Hospital Leiden, Leiden, The Netherlands.

He is a physiologist at the Academic Medical Center (AMC), University of Amsterdam and Erasmus Medical Center (EMC), Erasmus University Rotterdam, The Netherlands. His chair in Clinical Physiology at the AMC is dedicated to cardiovascular aspects of perioperative and intensive care medicine focused on clinical and fundamental aspects of circulation and oxygenation. He heads the Department of Translational Physiology at the AMC. The Department consists of a group of basic and clinical scientist doing laboratory and clinical research in sepsis, shock, resuscitation, fluid therapy, blood transfusion, oxygen transport, microcirculation, mitochondria, acute kidney injury, and heart failure. Based on his engineering background, he conducts medical technology research related to the afore mentioned topics. Since 2007, he joined for $40 \%$ the Department of Intensive Care of the Erasmus Medical Center Rotterdam. He holds several patents, has an Hirsch-index of 63, and has authored over 600 peer reviewed papers.

Prof. Ince is past-president of the Dutch Physiological Society and pastpresident of the International Society of Oxygen Transport to Tissue. 


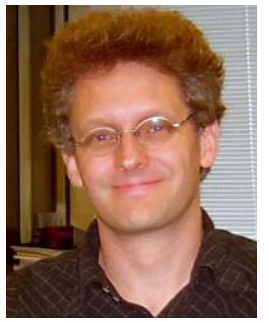

Johan G. Bosch (M'07) received the M.Sc. degree in electrical engineering from Eindhoven University of Technology, Eindhoven, The Netherlands, in 1985, and the Ph.D. degree from Leiden University Medical Center (LUMC), Leiden, The Netherlands, in 2006.

He performed ultrasound and image processing research at Erasmus University Rotterdam and Leiden University. From 1995 to 2005, he was an Assistant Professor and head of the Echocardiography Section at the Division of Image Processing (LKEB), Department of Radiology, LUMC. He is currently an Associate Professor and staff member with the Department of Biomedical Engineering, Thoraxcenter, Erasmus MC, Rotterdam. He is specialized in 2-D and 3-D echocardiographic image processing/analysis and transducer development. His main research interests are optimal border detection approaches, geometrical and statistical models, and anatomical and physical knowledge representations for border detection. He is currently leader of projects on 3-D segmentation and 3-D ultrasound guidance in electrophysiology and participates in several projects, e.g., on 3-D transducer development, 2-D and 3-D carotid imaging, and 3-D TEE imaging.

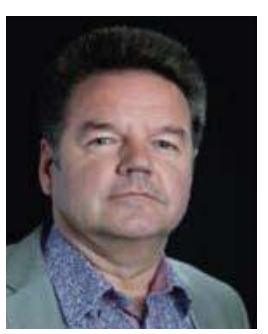

Antonius F. W. van der Steen (M'94-SM'03F'13) received the M.Sc. degree in applied physics from the Delft University of Technology, Delft, The Netherlands, in 1989, and the Ph.D. degree in medical sciences from the University of Nijmegen, Nijmegen, The Netherlands, in 1994.

From 1994 to 1996, he was a Senior Scientist with the Laboratory for Experimental Echocardiography, Thoraxcenter, Erasmus University Medical Center (MC), Rotterdam, The Netherlands, where he has been the Head of the Laboratory for Experimental Echocardiography since 1997. Since 2002, he has been a Full Professor and the Head of Biomedical Engineering with Erasmus University MC. Since 2013, he has been a Full Professor of Applied Physics with the Delft University of Technology. Since 2013, he has also been an Honorary Visiting Professor with the Chinese Academy of Sciences, Beijing, China, and with the Shenzhen Institute of Advanced Technologies, Shenzhen, China. His current research interests include vulnerable plaque detection, intravascular imaging, biomechanics, ultrasound contrast agents, and transducer design for special applications.

Prof. van der Steen is currently a member of the Royal Dutch Academy of Science and the Netherlands Academy of Technology and Innovation (i.e., Dutch Academy of Engineering). He is a Fellow of the European Society of Cardiology. He is the 2000 NWO PIONIER Technical Sciences (Best Researcher of the Netherlands in technical science under 40) and the 2007 Simon Stevin Master (Best Researcher in translational research).

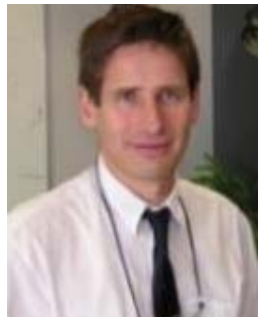

Nico de Jong (A'97-M'09) graduated from Delft University of Technology, The Netherlands, in 1978. He received the M.Sc. degree in physics specialised in the field of pattern recognition, and the Ph.D. degree for acoustic properties of ultrasound contrast agents, in 1993

Since 1980, he is staff member of the thoraxcenter of the Erasmus Medical Center, Rotterdam, The Netherlands. In 2003, he became part-time professor at the University of Twente. Over the last 5 years, he has given more than 30 invited lectures and has given numerous scientific presentations for international industries. He teaches on Technical Universities and the Erasmus MC. He has been Promotor of 21 $\mathrm{Ph} . \mathrm{D}$. students and is currently (co)-supervising $11 \mathrm{Ph} . \mathrm{D}$. students. He has published 260 peer reviewed articles and has been PI and Workpackage leader in European and Dutch projects. His H-factor is 46 (Web of Science) and he has acquired more than $6 \mathrm{ML}$ as PI or co-PI since 2010. Since October 2011, he is professor in Molecular Ultrasonic Imaging and Therapy at the Erasmus MC and the Technical University of Delft and since December 2015 (parttime) head of the department Acoustical Waveform Imaging at the Technical University in Delft.

Dr. de Jong is organizer of the annual European Symposium on Ultrasound Contrast Imaging, held in Rotterdam and attended by approximately 175 scientists from universities and industries all over the world. He is on the safety committee of WFUMB (World Federation of Ultrasound in Medicine and Biology), associate editor of UMB and has been guest editor for special issues of different journals.

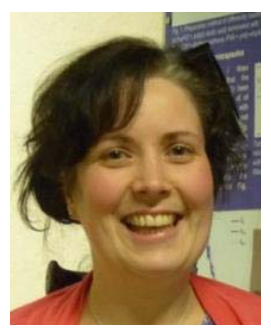

Klazina Kooiman (M'12) studied biopharmaceutical sciences at Leiden University, the Netherlands, and received the M.Sc. (cum laude) degree specializing in pharmaceutical technology. From 2005 to 2010, she was a Ph.D. student in the Department of Biomedical Engineering of the Thorax Centre, Erasmus MC, the Netherlands. She received the Ph.D. degree on the topic of ultrasound contrast agents for therapy in 2011.

At EFUSMB 2011 in Vienna, she won the young investigator award. She was awarded the ICIN Fellowship 2012, which enabled her to perform research for seven months in 2012 to 2013 in the lab of Professor F. S. Villanueva, director of the Center for Ultrasound Molecular Imaging and Therapy at UPMC, Pittsburgh, PA, USA. In 2014, she acquired the prestigious VENI grant from the Dutch Technology Foundation STW, which is part of the Netherlands Organisation for Scientific Research (NWO) and in 2015 the Erasmus MC fellowship. The grants focus on ultrasound contrast agents for vascular and bacterial drug delivery, respectively. She is currently assistant professor and head of the Therapeutic Ultrasound Contrast Agent group within the Department of Biomedical Engineering of the Thorax Centre, Erasmus MC, focusing on using ultrasound contrast agents for drug delivery and molecular imaging. She organizes the annual European Symposium on Ultrasound Contrast Imaging, held in Rotterdam, which is attended by approximately 175 scientists from universities and industries all over the world. 